\title{
The effects of hypoxia on mitochondrial function and metabolism in gastric cancer cells
}

\author{
Jun Jiang', Yuan Jiang' ${ }^{2}$, Yao-Gang Zhang ${ }^{2}$, Tao Zhang ${ }^{3}$, Jian-Hua Li ${ }^{2}$, Deng-Liang Huang ${ }^{2}$, Jing Hou ${ }^{2}$, \\ Mei-Yuan Tian', Li Sun ${ }^{2}$, Xiao-Ming Su ${ }^{3}$, Yun Dong ${ }^{3}$, Yan-Yan Ma ${ }^{2,4}$ \\ ${ }^{1}$ Department of Oncology, Affiliated Hospital of Qinghai University, Xining, China; ${ }^{2}$ Central Laboratory of Qinghai University Affiliated Hospital, \\ Affiliated Hospital of Qinghai University, Xining, China; ${ }^{3}$ Graduate School of Qinghai University, Qinghai University, Xining, China; ${ }^{4}$ Department \\ of Scientific Research Office, Affiliated Hospital of Qinghai University, Xining, China \\ Contributions: (I) Conception and design: J Jiang, Y Jiang, YY Ma; (II) Administrative support: None; (III) Provision of study materials: T Zhang, J \\ Hou; (IV) Collection and assembly of data: XM Su, Y Dong; (V) Data analysis and interpretation: YG Zhang, L Sun; (VI) Manuscript writing: All \\ authors; (VII) Final approval of manuscript: All authors. \\ Correspondence to: Yan-Yan Ma, PhD. Department of Scientific Research Office, Affiliated Hospital of Qinghai University, Tongren Road 29, Xining, \\ OH 810000, China. Email: mayanyan_research@qhu.edu.cn.
}

Background: A number of studies have found that metabolic disorders are the characteristic manifestations of tumor cells. However, the effects of hypoxic environment on mitochondrial function and glucose metabolism of tumor cells were still unclear. The study wanted to explore the regulatory mechanism of hypoxic environment on mitochondrial function and metabolism in gastric cancer cells.

Methods: The animal model of gastric cancer and MKN45 were treated in a hypoxic environment. Mitochondrial membrane potential and reactive oxygen species (ROS) levels were analyzed by flow cytometry, qPCR was used to detect the expression levels of glycose metabolism key enzymes, damage repair genes and mitochondrial DNA (mtDNA) copy numbers in gastric cancer.

Results: Compared with 2,000 m normal gastric cancer tissue, the decreased of mitochondrial membrane potential and the production of ROS reduced, the expressions of glucose metabolism genes [the M1 isoform of Hexokinase (HK1), pyruvate kinase (PKM), Succinate dehydrogenase (SDHA), Glucose-6-phosphate dehydrogenase (G6PD)], homologous recombination repair gene (RAD51) and repair DNA double-stranded broken gene (ASTCT2) increased, and aerobic respiration reduced in gastric cancer cells. In the hypoxic environment, the decreased of mitochondrial membrane potential reduced, the production of ROS and mtDNA copies increased, HK1 expression increased, the expressions of SDHA, G6PD, RAD51 and ASCT2 decreased, and the aerobic respiration decreased.

Conclusions: Hypoxia plays an important role in maintaining mitochondrial functions in gastric cancer cells by promoting glycolysis and inhibiting mitochondrial aerobic respiration capacity.

Keywords: Gastric cancer; hypoxia; mitochondrial function; aerobic respiration

Submitted Jul 25, 2020. Accepted for publication Dec 11, 2020.

doi: $10.21037 /$ tcr-20-2598

View this article at: http://dx.doi.org/10.21037/tcr-20-2598

\section{Introduction}

Gastric cancer is the most common malignancy of the digestive tract. There was $1,000,000$ new cases of gastric cancer worldwide accounting for $5.7 \%$ of new cancer cases, and 783,000 gastric cancer deaths accounting for $8.2 \%$ of cancer deaths in 2018 (1). According to the 2019 China
Cancer Report, the incidence of new gastric cancer is $10.26 \%$ and the mortality rate of gastric cancer is $12.45 \%$. In addition, gastric cancer was the second incidence and third mortality in 2015. In China, the detection rate and diagnosis rate of early gastric cancer were less than $10 \%$, and most patients were already in the advanced stage of 
gastric cancer or the tumor has metastasized. The 5-year survival rate for patients with advanced gastric cancer is still less than $15 \%$ after treatment (2). Therefore, gastric cancer has become a serious threat to public health diseases.

Hypoxia is a characteristic of tumor cells which can lead to tumor progression, invasion, and metastasis $(3,4)$. Metabolic disorders are the characteristic features of malignant tumor metabolism and promote tumor development (5). Warburg first found that tumor cells still convert glucose into lactic acid for anaerobic metabolism even in the presence of sufficient oxygen in 1956 (6). Hypoxic environment significantly inhibits oxidative phosphorylation and increases the expression of key enzymes or important molecules in glycolysis, thus promoting glycolysis metabolism (7). Promoting glycolysis accelerated the progression of tumor cells and inhibiting glycolysis reduced the metastasis of tumors (8). Hexokinase (HK), pyruvate kinase M1 (PKM1), Succinate dehydrogenase complex A (SDHA), glucose-6phosphate dehydrogenase (G6PD) are the key enzymes to regulate glycolysis, tricarboxylic acid cycle (TCA cycle) and pentose phosphate pathway. Current studies have found that HK1 and PKM2 is highly expressed in tumor cells, which can enhance the Warburg reaction to promote tumor growth, and related to the staging, lymphatic metastasis and prognosis of gastric cancer (9). 6-diphosphatase 1 (FBP1), a key enzyme in gluconeogenesis, was found to inhibit glycolysis of cancer cells (10). Lung cells were found to change the type of cell energy metabolism from oxidative phosphorylation to glycolysis in a hypoxic environment to reduce oxidative stress and promote cell proliferation and invasion (11). Therefore, clarifying the activity of key enzymes of glucose metabolism in gastric cancer cells in hypoxic environment may provide a new research direction for anticancer treatment.

The study will explore the changes of mitochondrial metabolic function and the level of glucose metabolism enzymes in gastric cancer cells in the hypoxic environment from the animal and cell levels, so as to provide a new treatment strategy for clinical cancer treatment. We present the following article in accordance with the ARRIVE reporting checklist (available at http://dx.doi.org/10.21037/ tcr-20-2598).

\section{Methods}

\section{Gastric cancer model}

C57B/L6 mice (W: 18-22 g) were bought by Qinglongshan
Animal Breeding Farm, Jiangning District, Nanjing City (license number: SCXK2019-0002). The syringe sucked up gastric cancer cells, and then gastric cancer cells were injected into the wall of the mice's stomach. The gastric cancer model mice were fed for 2 months. Experiments were approved by the Ethics Committee of Affiliated Hospital of Qinghai University (P-SL-2017009) and animal operations conformed to measures for the Administration of Laboratory Animals in Qinghai Province.

\section{Cell culture}

The human gastric cancer lines MKN45 from the Cell Bank of the Chinese Academy of Sciences (Shanghai, China). MKN45 were respectively cultured in DMEM/F12 (1:1) and RPMI1640 supplemented with $10 \%$ fetal bovine serum at $37^{\circ} \mathrm{C}$ in a humidified incubator with $5 \% \mathrm{CO}_{2}$. For hypoxic condition, MKN45 were incubated in a hypoxic incubator containing $1 \% \mathrm{O}_{2}$, which was balanced by $\mathrm{CO}_{2}$ and nitrogen.

\section{Main instruments and reagents}

BD Celesta Flow Cytometer (FACS Celesta, BD, USA), fluorescent quantitative analysis system (Tissue FAXS-S Plus, Tissue Gnostics, Austria), cellular metabolism analysis system (Seahorse XFe96 Analyzer, Agilent, USA) and real-time PCR instrument (Light Cycler 480 II, Roche, Switzerland).

\section{Group of experimental animals}

C57B/L6 mice were randomly divided into normal group $(n=5)$ and gastric cancer group $(n=15)$, and the gastric cancer model is based on the method of TakeshiKuwata (12). The human gastric cancer cell line BGC823 was transplanted in situ into C56/b mice. Then different batches of mice in normal group and gastric cancer group were placed in animal decompression chamber (Yantai Ice Wheel High Pressure Oxygen Chamber Co, Ltd.) to simulate 2,000, 3,000, and 4,000 m altitude environments, lifting speed of $10 \mathrm{~m} / \mathrm{s}$, and each altitude was treated for 7 days. Normal-2,000 $\mathrm{m}$ and gastric cancer-2,000 $\mathrm{m}$ groups mice were kept in a feeding chamber.

\section{Analysis of mitochondrial membrane potential and ROS by the flow cytometry}

$0.01 \mathrm{~g}$ sample of fresh gastric cancer tissue was put in PBS 
buffer to create a single cell suspension. The suspension was centrifuged at $400 \mathrm{~g}$ for $10 \mathrm{~min}$, and the gastric cancer cell suspension was resuspended to a concentration of $5 \times 10^{5}$ cells/mL. MKN45 was collected in normal and anoxic conditions for flow cytometry analysis, and $5 \times 10^{5}$ cells were used to perform each assay in triplicate.

Reactive oxygen species (ROS) assay kit (Beyotime, S0033) and mitochondrial membrane potential assay kit (Becton, S551302) were respectively utilized to examine ROS and mitochondrial membrane potential of each group of cells treated with different oxygen concentrations.

Real-time qPCR analysis of HK1, PKM, SDHA, G6PDCT-2, RAD51, ASCT-2, and mtDNA expression in gastric tissue

Filtered distilled water was used as the no-template control. Template without reverse transcription was used to check for contamination with genomic DNA. Real-time qPCR was used to measure HK1, PKM, SDHA, G6PDCT-2, RAD51, ASCT-2, mtDNA and TFAM mRNA expression. The reaction system is $20 \mu \mathrm{L}$. Reaction conditions: predenaturation at $95^{\circ} \mathrm{C}$ for $10 \mathrm{~min}$, denaturation at $94^{\circ} \mathrm{C}$ for $60 \mathrm{~s}$, annealing extension at $60{ }^{\circ} \mathrm{C}$ for $34 \mathrm{~s}$ (fluorescence signal is collected in this step), 40 cycles (LightCycler 480 II).

\section{The seaborse analysis mitochondrial respiratory function}

MKN45 cells were seeded in XF 96-well and the number of cells per well is $1.5 \times 10^{4}$; in this way two 96 -well plates were prepared and respectively incubated in normoxic and hypoxic conditions for 24 hours prior to cell metabolic analysis. The XF Cell Mito Stress Test kit (Cat\# 103015100) was used assay mitochondrial metabolism of the cells treated with different lactate and oxygen concentrations.

\section{Statistical analysis}

Statistical analysis was performed using the SPSS 19.0 statistical software program. The normal distribution data were expressed as mean \pm standard deviation, a Student's t unpaired test was performed between the two groups, and one-way analysis of variance (ANOVA) was used to evaluate the intergroup differences. The level of significance was determined as a probability level of less than 0.05 .

\section{Results}

High altitude low oxygen environment can reduce the damage of mitochondrial function and protect mitochondria from damage in gastric cancer rats

Effects of high altitude hypoxia environment on mitochondrial membrane potential and ROS in gastric cancer tissue

The gastric of normal and model mice showed in Figure $1 \mathrm{~A}$. The decreased mitochondrial membrane potential and ROS production in gastric cancer tissues were significantly higher than those in the control group at the same altitude (Figure $1 B, C, D, E$ ). The decreased level of mitochondrial membrane potential in gastric cancer-2,000 $\mathrm{m}$ group was significantly higher than that in 3,000 and $4,000 \mathrm{~m}$ gastric cancer groups $(\mathrm{P}<0.001, \mathrm{P}=0.0011)$ (Figure 1C). The production of ROS in gastric cancer groups was higher than that in normal groups, and the level of ROS reduced in gastric cancer groups as the altitude increasing (Figure 1D). The results suggested that hypoxia could play a protective role by inhibiting the decrease of mitochondrial membrane potential and the increase of ROS production in gastric cancer.

\section{Effects of high altitude hypoxic environment on} mtDNA expression in gastric tissue

The standard curve is shown in Figure $2 A, B$ is the sample amplification curve, and Figure $2 C$ is standard product amplification curve. The expression of mtDNA reduced in normal groups as the altitude increasing. The expression of mtDNA in gastric cance-2,000 $\mathrm{m}$ group was higher than that in gastric cancer- $3,000 \mathrm{~m}$ group $(\mathrm{P}<0.001)$, and the expression of mtDNA in gastric cancer-4,000 $\mathrm{m}$ group was higher than that in gastric cancer-3,000 $\mathrm{m}$ group $(\mathrm{P}<0.001)$ (Figure 2D).

\section{Effects of high altitude hypoxic environment on} enzymes related to mitochondrial metabolism in gastric cancer tissue

The expression of HK1, PKM, SDHA, G6PD, AST2, RAD51 decreased with the elevation of altitude in normal groups (Figure $3 A, B, C, D, E, F$ ), and the expression of PKM, G6PD decreased with the elevation of altitude in gastric cancer groups $(\mathrm{P}<0.001)$ (Figure $3 B, D)$. HK1, SDHA, AST2 expression in gastric cancer-3,000 $\mathrm{m}$ group was higher than that in gastric cancer-2,000 $\mathrm{m}$ and gastric cancer-4,000 
A


C


D

$2000 \mathrm{~m}$ $3000 \mathrm{~m}$
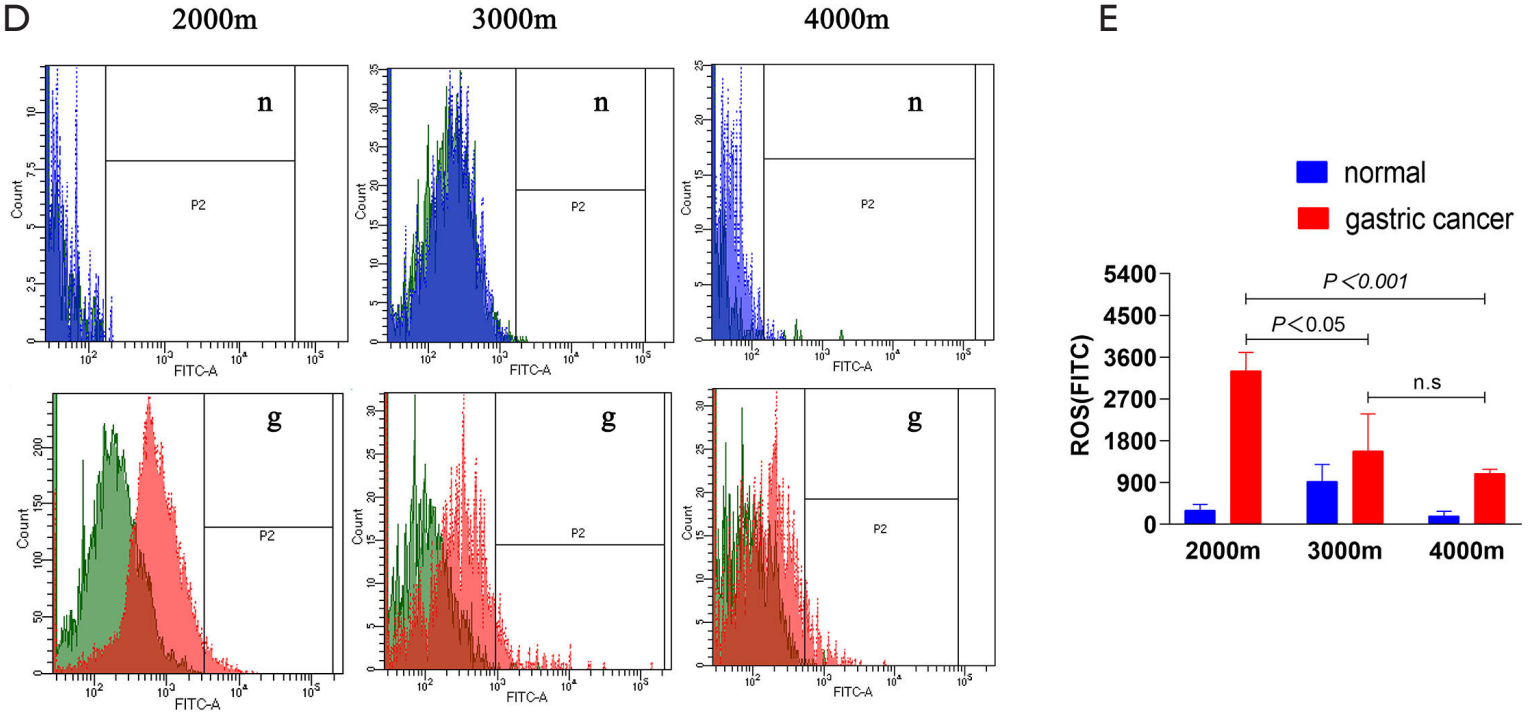

Figure 1 Results of gastric tissue mitochondrial membrane potential and ROS. (A) Normal and model mice gastric. (B) The results of mitochondrial membrane potential. (C) The results of mitochondrial membrane potential. (D) The results of ROS. (E) The results of ROS. Four samples per time point and 3 sample tubes per sample for flow cytometry. P3 represents a decrease in mitochondrial membrane potential. $\mathrm{P} 2$ represents the generation of ROS. 


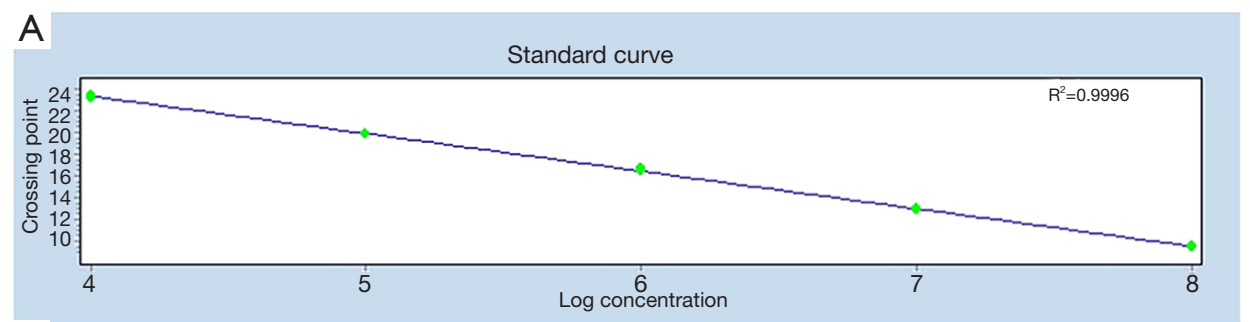

D
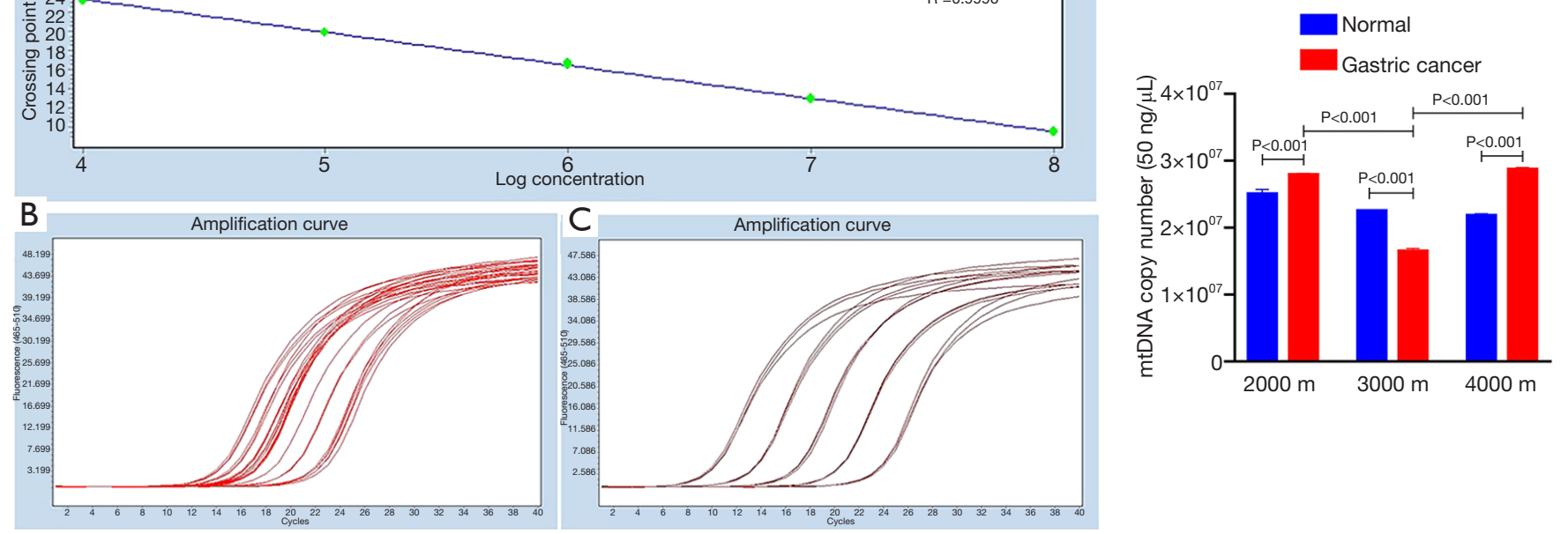

Figure 2 Results of gastric tissue mtDNA. The recombinant plasmid of cytochrome B (Cyt-B) gene sequence encoded by mtDNA of rats was constructed as a standard, and the Cyt-B gene in mitochondria of rat stomach tissues was quantified by real-time quantitative PCR, so as to achieve the absolute quantitative determination of mtDNA content. (A) The standard curve. (B) The sample amplification curve. (C) Standard product amplification curve. (D) The result of gastric tissue mtDNA.
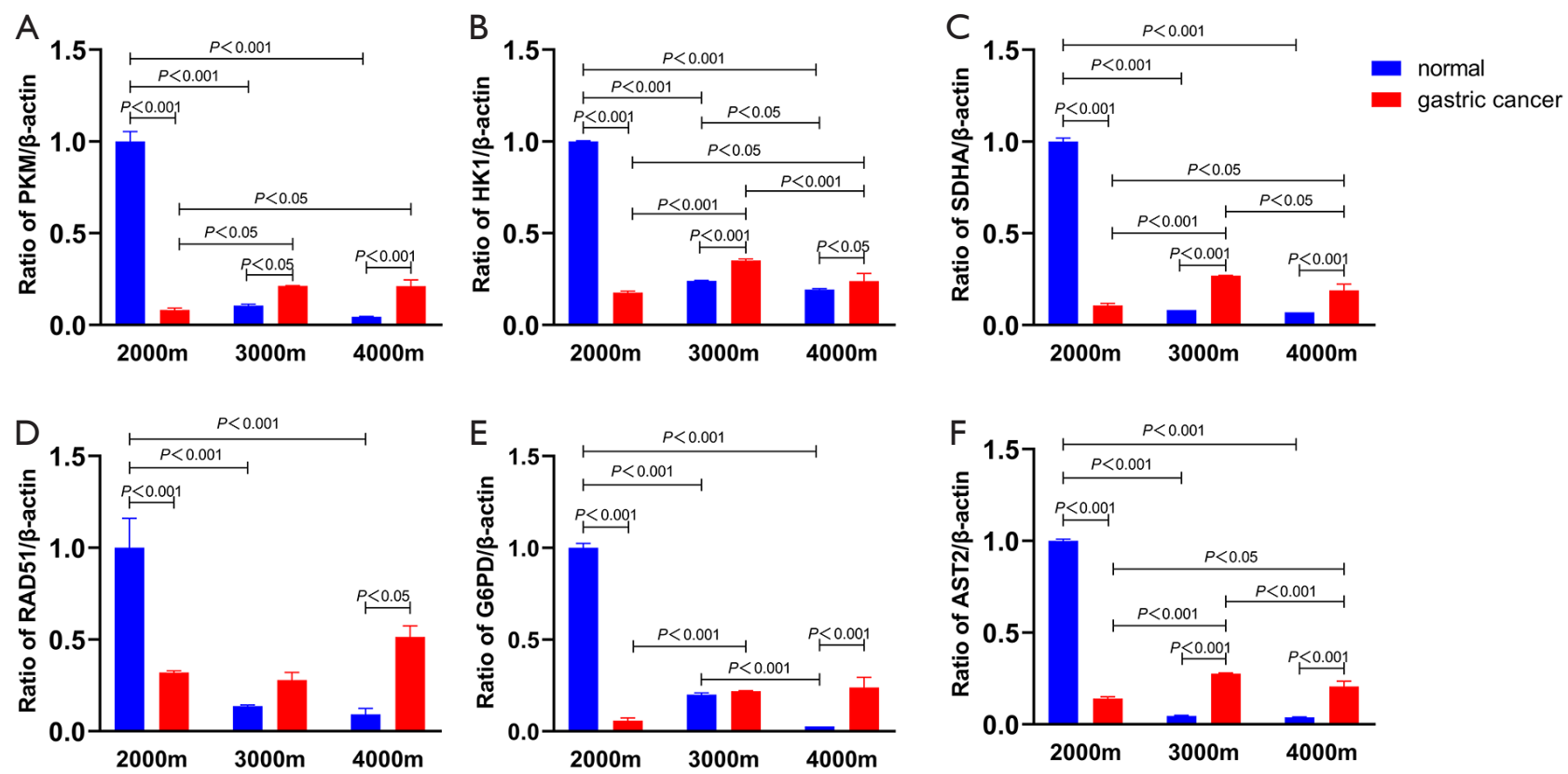

Figure 3 The level of HK1, PKM, SDHA, G6PDCT-2, RAD51 and ASCT-2 mRNA in gastric tissue. (A) The level of HK1 mRNA. (B) The level of PKM mRNA. (C) The level of SDHA mRNA. (D) The level of G6PD mRNA; (E) The level of AST2 mRNA. (F) The level of mRNA. 
A

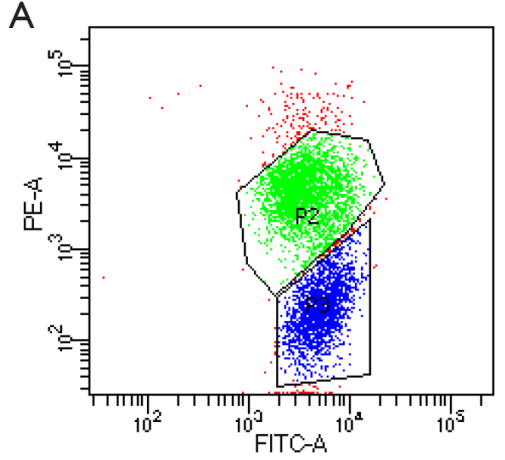

D

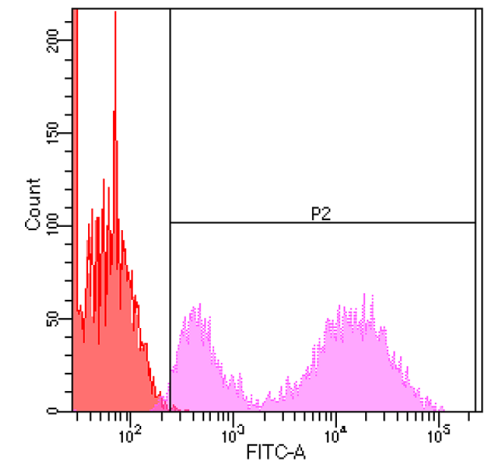

B

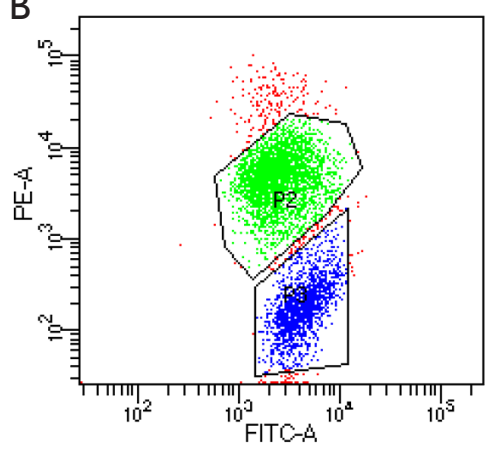

$\mathrm{E}$

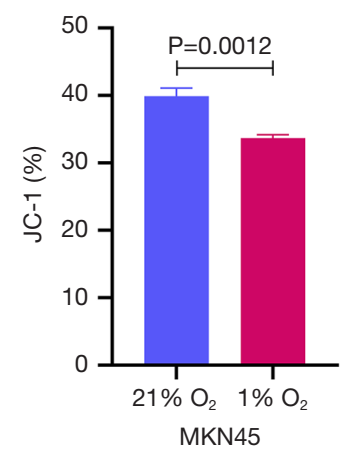

C



$\mathrm{F}$

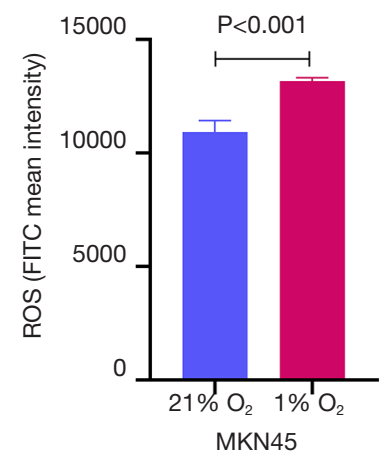

Figure 4 Results of hypoxia mitochondrial membrane potential and ROS in MKN45. (A,B) The results of MKN45 mitochondrial membrane potential. (C,D) Present the results of MKN45 ROS. (E) The statistical of MKN45 mitochondrial membrane potential. (F) The statistical of MKN45 ROS.

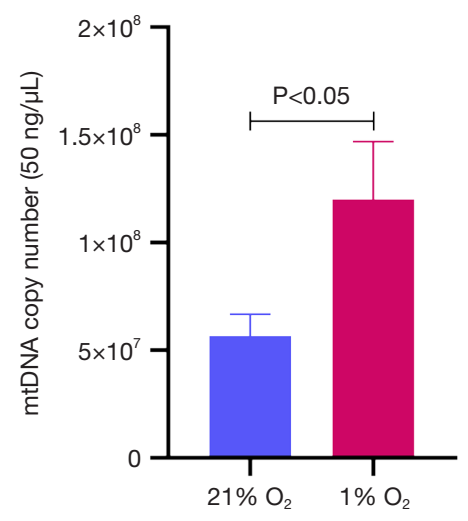

Figure 5 Results of MKN45 mtDNA.

$\mathrm{m}$ groups. The results suggest that hypoxia can promote glycolysis, tricarboxylic acid cycle and pentose phosphate pathway, and also promote the expression of damage repair genes in gastric cancer.

\section{Effects of high altitude hypoxia environment on} mitochondria functions of MKN45

Analysis of hypoxia mitochondrial membrane potential and ROS in MKN45

The results of mitochondrial membrane potential were shown in Figure $4 A, B$, and ROS production were shown in Figure $4 C, D$. The decreased mitochondrial membrane potential and ROS production in hypoxia condition were significantly lower than those in the normoxia condition $(\mathrm{P}=0.0012, \mathrm{P}<0.001)$ (Figure 4E,F). Hypoxia can inhibit the decrease of mitochondrial membrane potential and promote the production of ROS.

Effects of hypoxic environment on mtDNA expression in MKN45

The expression of mtDNA in hypoxia condition was higher than that in normoxia condition $(\mathrm{P}<0.001)$ (Figure 5). The results showed that hypoxia can promote the 
A



D

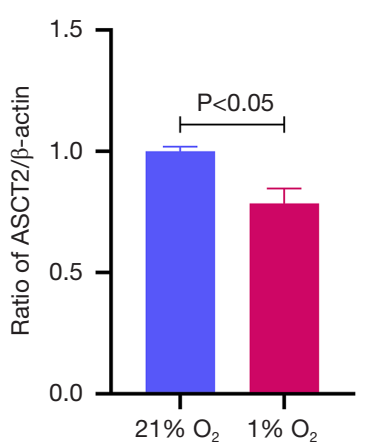

B

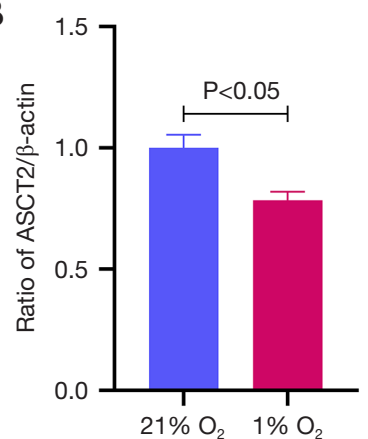

E



C

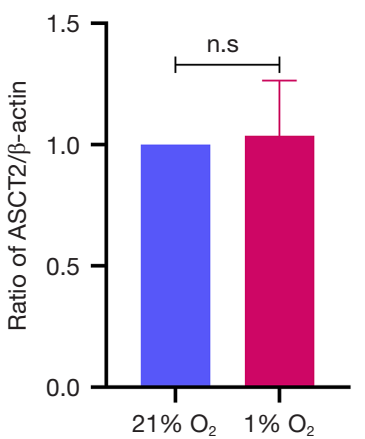

$\mathrm{F}$

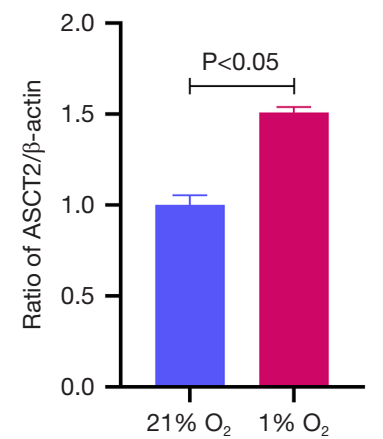

Figure 6 Results of HK1, PKM, SDHA, G6PDCT-2, RAD51 and ASCT-2 mRNA in MKN45. (A) The level of HK1 mRNA. (B) The level of SDHA mRNA. (C) The level of G6PD mRNA. (D) The level of AST2 mRNA. (E) The level of RAD5 mRNA. (F) The level of PKM mRNA.

expression of mtDNA.

Effects of hypoxic environment on enzymes related to mitochondrial metabolism in MKN45

The level of HK1 expression in hypoxia condition was higher than that in normoxia condition $(\mathrm{P}<0.05)$ (Figure 6A), SDHA, G6PD, AST2, RAD51 expressions in hypoxia condition was lower than that in normoxia condition $(\mathrm{P}<0.05)$ (Figure 6B,C,D,E), and no difference was found between the level of PKM mRNA in normoxia condition and hypoxia condition $(\mathrm{P}>0.05)$ (Figure $6 F)$. It suggests that hypoxia can change the metabolic pattern of glucose and promote glycolysis.

\section{Effects of hypoxic environment on mitochondrial respiratory function in $\mathrm{MKN} 45$}

Seahorse XFe 96 was used to detect cellular respiratory function. Mitochondrial stress test was shown in Figure $7 \mathrm{~A}$. The respiratory function of MKN45 at different oxygen concentrations was shown in Figure $7 B$. The proton leak (Figure 7C), ATP production (Figure 7D) and spare respiratory capacity (Figure $7 E$ ) in hypoxia group was declined than in normoxia group of the MKN45 $(\mathrm{P}<0.001$, $\mathrm{P}<0.001, \mathrm{P}<0.05)$.

\section{Discussion}

Gastric cancer is a leading cause of cancer-related deaths worldwide, especially in developing country (such as Asia, Eastern Europe) $(13,14)$. Hypoxia is a common feature of tumor cells and plays an important role in therapeutic resistance, recurrence and metastasis $(15,16)$. Metabolic disorders are the characteristic features of malignant tumor metabolism, which can promote tumor development (5). Exploring the role of hypoxia in gastric cancer cells may provide a new treatment for gastric cancer.

The glycolysis of gastric cancer cells was significantly higher than that of normal cells, and hypoxia also promotes glycolysis in gastric cancer cells $(17,18)$. Warburg found that the metabolize respiration of cancer cells was different from normal cells. Even if in plentiful oxygen, cancer cells prefer glycolysis for energy. HK, PKM, SDHA, G6PD are the key 
A
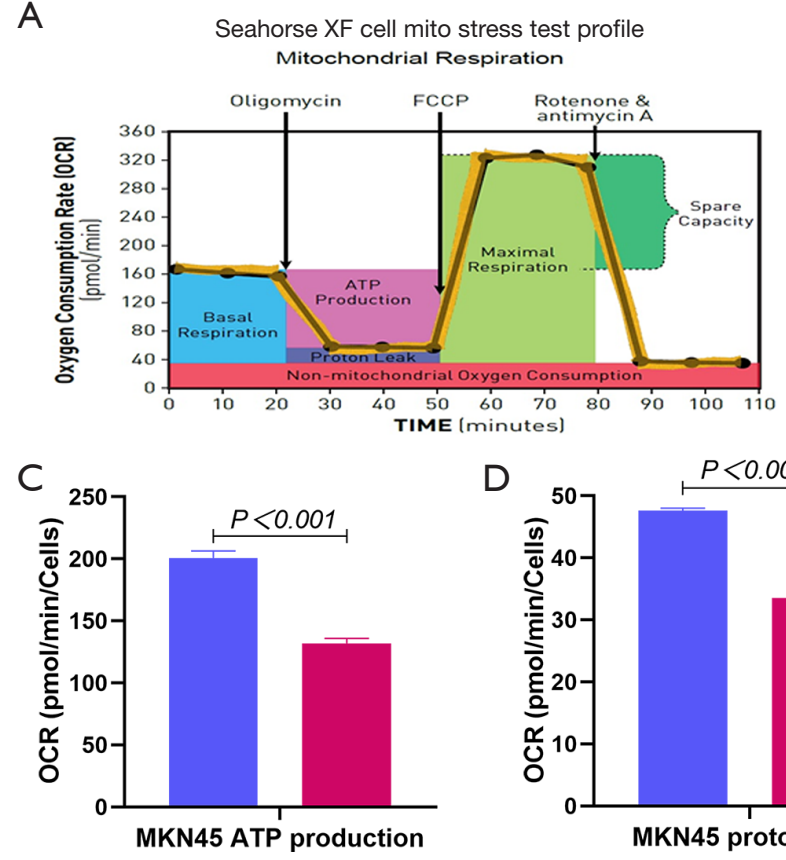

$\mathrm{D}$

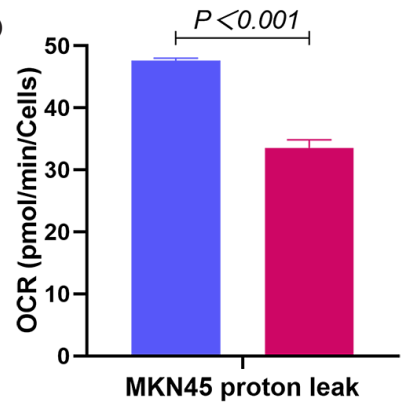

B
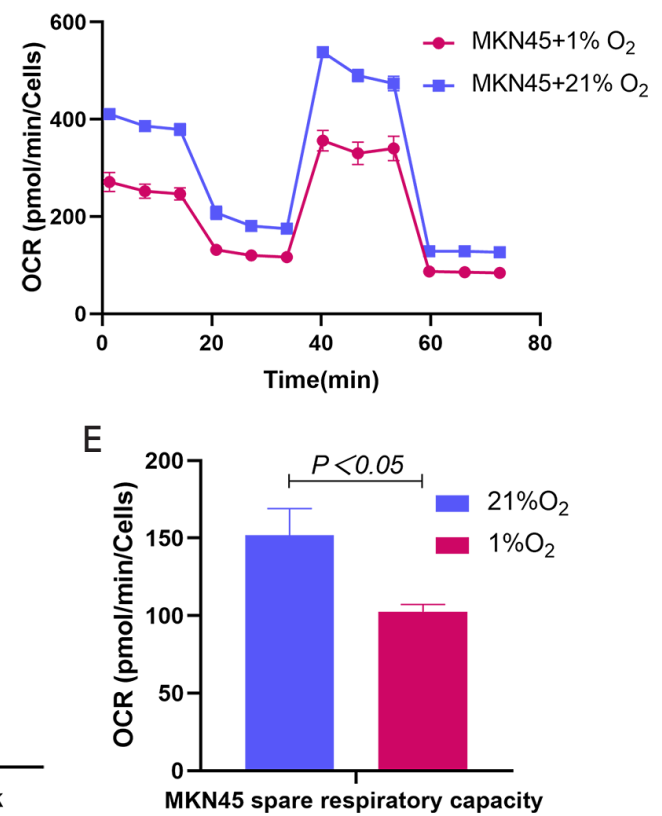

Figure 7 The seahorse analysis of the MKN45. (A) Mitochondrial stress test steps. (B) the O2 consumption rate (OCR) of MKN45 at different oxygen concentrations. (C) The proton leak. (D) ATP production. (E) Spare respiratory capacity. Pairwise comparison using independent sample $t$-test.

enzymes in glucose metabolism $(19,20)$. HK is a key enzyme that catalyzes the first step of the glycolysis pathway, which catalyze the conversion of glucose to glucose 6-phosphate (21). PKM catalyzes the conversion of phosphoenolpyruvate and ADP to pyruvate and ATP. SDHA catalyzed succinic acid to fumaric acid in tricarboxylic acid cycle, and G6PD catalyzed 6-phosphate glucose to 6-phosphate gluconic acid in pentose phosphate pathway. Previous studies found that the expression levels of HK1 and PKM2 in gastric cancer cells were increased $(9,22)$. Overexpression of PKM2 enhanced warburg effect of tumor and promoted the growth of tumor cells (23). Overexpression of PKM2 contributes to tumor progression, invasion and metastasis $(9,22)$. Inhibiting glycolysis and reducing energy supply can inhibit proliferation and promote apoptosis of tumor cells $(24,25)$. The study also found that the expression of HK1 and PKM increased in gastric cancer tissue. What is more, we found that the expression of G6PD and SDHA increased, and aerobic respiration reduced in gastric cancer tissue. That suggests that gastric cancer cells play a role in maintaining mitochondrial function by promoting glucose metabolism and inhibiting mitochondrial oxidative function.
Domestic researches have founded that hypoxic can promote the proliferation of breast cancer and lung cancer cells, enhance their invasion and metastasis ability, and promote the occurrence of epithelial to mesenchymal transformation (EMT) of cancer cells. Some studies have found that hypoxia can promote cell proliferation by increasing the expression of transforming growth factor, C-X-C Motif Chemokine Receptor 4 and Jumonji domain, containing 2B (26-28). Hypoxia can change the transcription and translation of genes related to DNA damage response and repair, leading to inhibit the recombination-mediated repair of double strand breaks in DNA. We also found that hypoxia can inhibit the expression of RAD51 and ASCT2, and HK1 expression was increased, G6PD and SDHA expression were decreased in MKN45. The results suggest that hypoxia can reduce mitochondrial aerobic respiration capacity and inhibit RAD51 and ASCT-2 expression, promote mtDNA expression and glycolysis process, which may play an important role in mitochondrial function in gastric cancer.

The present study had several limitations. First, we only studied MKN45, and we can increase other gastric cancer cell lines. Second, we found the effect of hypoxia 
on mitochondrial function, but the exact mechanism is not clear.

\section{Conclusions}

Hypoxia plays an important role in maintaining mitochondrial functions in gastric cancer cells by promoting glycolysis and inhibiting mitochondrial aerobic respiration capacity.

\section{Acknowledgments}

We thank the Qinghai province Research Key Laboratory for Echinococcosis.

Funding: This work was supported by Qinghai Science and Technology Foundation (No. 2017-ZJ-710) and Key Projec of Qinghai Provincial Health Commission (No. 2020-wjzd-03).

\section{Footnote}

Reporting Checklist: The authors have completed the ARRIVE reporting checklist. Available at http://dx.doi. org/10.21037/tcr-20-2598

Data Sharing Statement: Available at http://dx.doi. org/10.21037/tcr-20-2598

Conflicts of Interest: All authors have completed the ICMJE uniform disclosure form (available at http://dx.doi. org/10.21037/tcr-20-2598). The authors have no conflicts of interest to declare.

Etbical Statement: The authors are accountable for all aspects of the work in ensuring that questions related to the accuracy or integrity of any part of the work are appropriately investigated and resolved. Experiments were approved by the Ethics Committee of Affiliated Hospital of Qinghai University (P-SL-2017009) and animal operations conformed to measures for the Administration of Laboratory Animals in Qinghai Province.

Open Access Statement: This is an Open Access article distributed in accordance with the Creative Commons Attribution-NonCommercial-NoDerivs 4.0 International License (CC BY-NC-ND 4.0), which permits the noncommercial replication and distribution of the article with the strict proviso that no changes or edits are made and the original work is properly cited (including links to both the formal publication through the relevant DOI and the license). See: https://creativecommons.org/licenses/by-nc-nd/4.0/.

\section{References}

1. Bray F, Ferlay J, Soerjomataram I, et al. Global cancer statistics 2018: GLOBOCAN estimates of incidence and mortality worldwide for 36 cancers in 185 countries. CA Cancer J Clin 2018;68:394-424.

2. Edge SB, Compton CC. The American Joint Committee on Cancer: the 7th edition of the AJCC cancer staging manual and the future of TNM. Ann Surg Oncol 2010;17:1471-4.

3. Shida M, Kitajima Y, Nakamura J, et al. Impaired mitophagy activates mtROS/HIF-1 $\alpha$ interplay and increases cancer aggressiveness in gastric cancer cells under hypoxia. Int J Oncol 2016;48:1379-90.

4. Gilkes DM, Semenza GL, Wirtz D. Hypoxia and the extracellular matrix: drivers of tumour metastasis. Nat Rev Cancer 2014;14:430-9.

5. Ishikawa K, Takenaga K, Akimoto M, et al. ROSgenerating mitochondrial DNA mutations can regulate tumor cell metastasis. Science 2008;320:661-4.

6. Warburg O. On the origin of cancer cells. Science 1956;123:309-14.

7. Lu X, Kang Y. Hypoxia and hypoxia-inducible factors: master regulators of metastasis. Clin Cancer Res 2010;16:5928-35.

8. Maximchik P, Abdrakhmanov A, Inozemtseva E, et al. 2-Deoxy-D-glucose has distinct and cell line-specific effects on the survival of different cancer cells upon antitumor drug treatment. FEBS J 2018;285:4590-601.

9. Gao Y, Xu D, Yu G, et al. Overexpression of metabolic markers HK1 and PKM2 contributes to lymphatic metastasis and adverse prognosis in Chinese gastric cancer. Int J Clin Exp Pathol 2015;8:9264-71.

10. Zhang B, Qin Y, Shi S, et al. FBP1, A tumor suppressor and negative regulator of glycolysis, was epigenetically silenced in pancreatic cancer. Curr Signal Transduct Ther 2014;9:156-63.

11. Chae YC, Vaira V, Caino MC, et al. Mitochondrial Akt Regulation of Hypoxic Tumor Reprogramming. Cancer Cell 2016;30:257-72.

12. Kuwata T, Yanagihara K, Iino Y, et al. Establishment of Novel Gastric Cancer Patient-Derived Xenografts and Cell Lines: Pathological Comparison between Primary Tumor, Patient-Derived, and Cell-Line Derived 
Xenografts. Cells 2019;8:585.

13. Siegel RL, Miller KD, Jemal A. Cancer statistics, 2019.

CA Cancer J Clin 2019;69:7-34.

14. Jemal A, Bray F, Center MM, et al. Global cancer statistics. CA Cancer J Clin 2011;61:69-90.

15. Dekervel J, Hompes D, van Malenstein H, et al. Hypoxiadriven gene expression is an independent prognostic factor in stage II and III colon cancer patients. Clin Cancer Res 2014;20:2159-68.

16. Zhang WJ, Chen C, Zhou ZH, et al. Hypoxia-inducible factor-1 alpha Correlates with Tumor-Associated Macrophages Infiltration, Influences Survival of Gastric Cancer Patients. J Cancer 2017;8:1818-25.

17. Jiang Z, Yang H, Zhang X, et al. Histone deacetylase-1 as a prognostic factor and mediator of gastric cancer progression by enhancing glycolysis. Hum Pathol 2019;85:194-201.

18. Tang Z, Li L, Tang Y, et al. CDK2 positively regulates aerobic glycolysis by suppressing SIRT5 in gastric cancer. Cancer Sci 2018;109:2590-8.

19. Lincet H, Icard P. How do glycolytic enzymes favour cancer cell proliferation by nonmetabolic functions. Oncogene 2015;34:3751-9.

20. Yang W, Lu Z. Nuclear PKM2 regulates the Warburg effect. Cell Cycle 2013;12:3154-8.

21. Nilsson H, Lindgren D, Mandahl Forsberg A, et al. Primary clear cell renal carcinoma cells display minimal mitochondrial respiratory capacity resulting in pronounced sensitivity to glycolytic inhibition by 3-Bromopyruvate.

Cite this article as: Jiang J, Jiang Y, Zhang YG, Zhang T, Li JH, Huang DL, Hou J, Tian MY, Sun L, Su XM, Dong Y, Ma YY. The effects of hypoxia on mitochondrial function and metabolism in gastric cancer cells. Transl Cancer Res 2021;10(2):817-826. doi: 10.21037/tcr-20-2598
Cell Death Dis 2015;6:e1585.

22. Li H, Xu H, Xing R, et al. Pyruvate kinase M2 contributes to cell growth in gastric cancer via aerobic glycolysis. Pathol Res Pract 2019;215:152409.

23. Yuan S, Qiao T, Zhuang X, et al. Knockdown of the M2 Isoform of Pyruvate Kinase (PKM2) with shRNA Enhances the Effect of Docetaxel in Human NSCLC Cell Lines In Vitro. Yonsei Med J 2016;57:1312-23.

24. Guo X, Zhang X, Wang T, et al. 3-Bromopyruvate and sodium citrate induce apoptosis in human gastric cancer cell line MGC-803 by inhibiting glycolysis and promoting mitochondria-regulated apoptosis pathway. Biochem Biophys Res Commun 2016;475:37-43.

25. Granchi C, Fancelli D, Minutolo F. An update on therapeutic opportunities offered by cancer glycolytic metabolism. Bioorg Med Chem Lett 2014;24:4915-25.

26. Noda S, Yashiro M, Nshii T, et al. Hypoxia upregulates adhesion ability to peritoneum through a transforming growth factor-beta-dependent mechanism in diffuse-type gastric cancer cells. Eur J Cancer 2010;46:995-1005.

27. Oh YS, Kim HY, Song IC, et al. Hypoxia induces CXCR4 expression and biological activity in gastric cancer cells through activation of hypoxia-inducible factor-1 $\alpha$. Oncol Rep 2012;28:2239-46.

28. Kim JG, Yi JM, Park SJ, et al. Histone demethylase JMJD2B-mediated cell proliferation regulated by hypoxia and radiation in gastric cancer cell. Biochim Biophys Acta 2012;1819:1200-7. 